\title{
Immunodiagnosis of Snake Bite
}

\author{
B. M. GREENWOOD, D. A. WARRELL, N. MCD. DAVIDSON, L. D. ORMEROD, H. A. REID
}

British Medical fournal, 1974, 4, 743-745

\section{Summary}

Management of a patient with snake bite is influenced by the nature of the offending snake. Species diagnosis based on the patient's history and physical signs is often unreliable and the possibility of making a species diagnosis by immunological means has therefore been investigated. Wound aspirates, blister fluids, sera, and urine samples from patients with snake bite were examined for the presence of species-specific venoms using immunodiffusion. A positive species diagnosis was made in 40 out of 101 patients. Immunodiagnosis was especially successful in patients bitten by the puff adder, Bitis arietans, and the African spitting cobra, Naja nigricollis. A higher success rate could probably be achieved using more specific antisera and more sensitive assay techniques.

\section{Introduction}

The rational management of patients with snake bite is facilitated by identification of the offending snake. It is often impossible, however, to establish an accurate species diagnosis on the basis of the patient's history and physical signs. Many clinical studies of snake bite include patients bitten by unidentified species. We have therefore investigated the possibility of making a species diagnosis in patients with snake bite by detection of specific venoms in body fluids. This technique was first described by Muelling et al. (1957) and has been used successfully in animals (Trethewie and Thomas, 1969). We describe here the results of immunodiagnosis in 101 patients with snake bite.

\section{Patients and Methods}

A total of 101 patients with snake-bite poisoning presenting at Ahmadu Bello University Teaching Hospital, Zaria, or Gombe or Kaltungo General Hospitals, were studied. In nine patients a species diagnosis of bites by Bitis arietans (puff adder), Causus maculatus (night adder), and Naja nigricollis (spitting cobra) was made when patients brought the snake that had bitten them. A diagnosis of Echis carinatus (carpet viper) bite was made in 66 patients bringing this snake or having incoagulable blood or both. Dispholidus typus (boomslang) is the only other snake found in northern Nigeria whose venom makes blood incoagulable, but human bites by this species are very rare except in snake handlers.

Samples.-Tissue fluid was aspirated from the site of the bite in 74 patients using a 21 -gauge needle, and adjacent blisters were aspirated in 13 patients. Serum and urine were collected

Department of Medicine, Ahmadu Bello University, Zaria, Nigeria B. M. GREENWOOD, M.D., M.R.C.P., Senior Lecturer in Medicine D. A. WARRELL, D.M., M.R.C.P., Consultant Physician (Present address: Hammersmith Hospital, London $W 12$ OHS) N. MCD. DAVIDSON, B.M., M.R.C.P., Senior Lecturer in Medicine
L. D. ORMEROD, M.B., D.T.M.\&H., Medical Registrar

Liverpool School of Tropical Medicine, Liverpool L3 5QA H. A. REID, M.D., F.R.C.P., Senior Lecturer in Tropical Medicine on admission to hospital and, together with wound aspirates, stored at $-20^{\circ} \mathrm{C}$ until tested. Urine samples were concentrated fiftyfold to a hundredfold by negative pressure dialysis using a US/UF microconcentrator (Biomed Instruments Inc.).

Antisera.-Thirteen species of snakes potentially venomous to man were collected by us in the savanna region of northern Nigeria. Many of these species are uncommon and in human snake bites in the region we identified only the following: $N$. nigricollis, $N$. haje (Egyptian cobra), E. carinatus, $C$. maculatus, Atractaspis dahomeyensis (burrowing viper), and $A$. mocrolepidota (burrowing viper). Specific antisera were raised in rabbits to venoms of locally obtained $N$. nigricollis, $N$. haje, $N$. melanoleuca, B. arietans, E. carinatus, and C. maculatus. Relatively specific antisera were obtained on direct immunization and monospecific sera were prepared by absorptions with appropriate freeze-dried venoms. Insufficient venom was obtained from snakes of the Atractaspis group or from D. typus to raise antisera but it was shown that these venoms did not cross-react with the specific antisera prepared as above. The following schedule was found to give good precipitating antisera without killing the immunized rabbits: an initial intramuscular injection of $100 \mu \mathrm{g}$ of venom in complete Freund's adjuvants was followed four weeks later by an injection of $200 \mu \mathrm{g}$ of venom in incomplete adjuvant. Two weeks later $1 \mathrm{mg}$ of venom was given intravenously. Serum was collected after a further fortnight.

Immunodiffusion and Immunoelectrophoresis.-Immunodiffusion was carried out in $1.5 \%$ agar in TRIS-barbital buffer $\mathrm{pH} 8.8$. Immunodiffusion wells were $3 \mathrm{~mm}$ in diameter and $5 \mathrm{~mm}$ apart. Plates were read after 48 hours incubation and reread after washing and treatment with $1 \%$ tannic acid for 10 minutes. Strong reactions were sometimes apparent after only six hours incubation, but weak lines were sometimes visible only after tannic-acid treatment. Counter-current immunoelectrophoresis was carried out in $0.75 \%$ agarose using a discontinuous TRIS-barbital buffer system (Greenwood and Whittle, 1974). Slides were read immediately after a 60-minute run and reread 12 hours later when faint reactions had sometimes developed.

\section{Results}

The results obtained on immunodiffusion of samples from 75 patients with a known species diagnosis and from a group of 26 patients in whom the biting snake could not be identified are shown in the table. Typical results are shown in the photo-

Detection of Venom by Immunodiffusion in Biological Fluids of Patients with Snake Bite. Results are Numbers of Samples Positive out of Numbers Tested

\begin{tabular}{|c|c|c|c|c|c|}
\hline \multirow{2}{*}{$\begin{array}{l}\text { Snake } \\
\text { Species }\end{array}$} & \multicolumn{5}{|c|}{ Body Fluid } \\
\hline & $\begin{array}{c}\text { Wound } \\
\text { Aspirate }\end{array}$ & $\begin{array}{c}\text { Blister } \\
\text { Aspirate }\end{array}$ & Serum & $\begin{array}{c}\text { Concentrated } \\
\text { Urine }\end{array}$ & $\begin{array}{c}\text { Any } \\
\text { Specimen }\end{array}$ \\
\hline $\begin{array}{l}\text { B. arietans } \\
\text { C. maculatus } \\
\text { E. carinatus } \\
N . \text { nigricollis } \\
\text { Unknown }\end{array}$ & $\begin{array}{c}1 / 2 \\
0 / 2 \\
16 / 46 \\
2 / 4 \\
8 / 20\end{array}$ & $\begin{array}{l}1 / 1 \\
0 / 1 \\
5 / 7 \\
1 / 1 \\
2 / 3\end{array}$ & $\begin{array}{l}0 / 2 \\
0 / 2 \\
0 / 56 \\
0 / 4 \\
0 / 20\end{array}$ & $\begin{array}{l}2 / 2 \\
0 / 2 \\
3 / 43 \\
5 / 5 \\
9 / 24\end{array}$ & $\begin{array}{c}2 / 2 \\
0 / 2 \\
22 / 66 \\
5 / 5 \\
11 / 26\end{array}$ \\
\hline Total & $27 / 74$ & $9 / 13$ & $0 / 84$ & $19 / 76$ & $40 / 101$ \\
\hline
\end{tabular}

graph. Venom was detected in all patients bitten by $B$. arietans or $N$. nigricollis but not in all those bitten by $E$. carinatus. A positive immunodiagnosis was made in 11 out of 26 patients in whom the identity of the biting snake was completely un- 

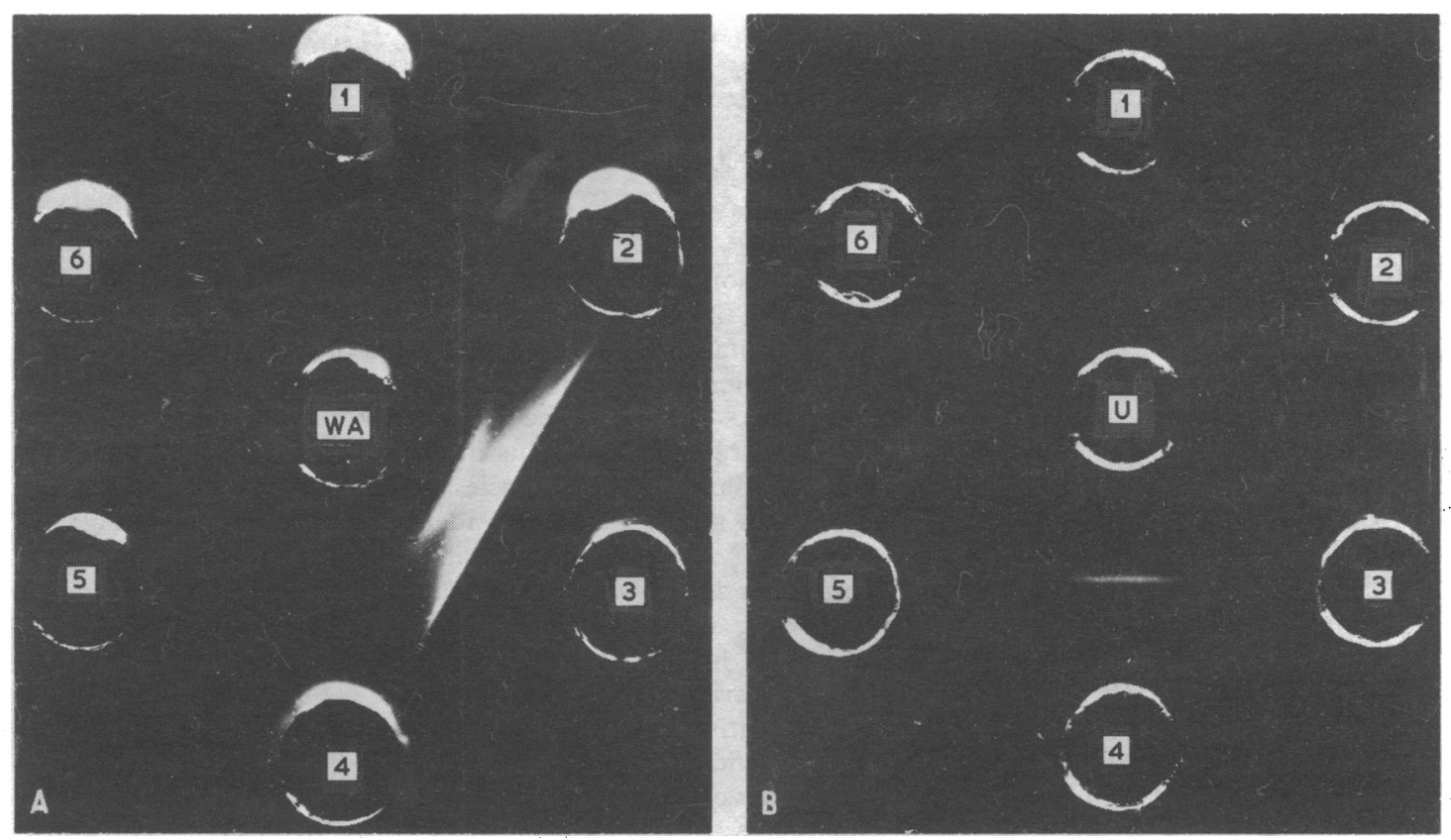
serum to $C$. maculatus, (2) antiserum to $E$. carinatus, (3) antiserum to $B$ arietans, (4) antiserum to $B$. arietans, collis, (5) antiserum to nigricollis, (5) antiserum to $N$ N. malenoleuca.

known (Naja species in seven, $B$. arietans in two, and $E$. carinatus in two). Samples from seven patients known to have $N$. nigricollis bites were tested against specific antisera to $N$. nigricollis, $N$. haje, and $N$. melanoleuca. Samples from five of the patients gave a precipitin line with only $N$. nigricollis antiserum, and the other two reacted with all three antisera.

One-third of wound aspirates and two-thirds of blister aspirates gave positive results. Urine samples were positive in patients bitten by $N$. nigricollis and $B$. arietans but were not often positive in patients bitten by $E$. carinatus. No sera were positive. One serum and two wound aspirates gave a nonspecific reaction with all antisera. Seven patients with venom in the urine were retested 24-48 hours after the administration of antivenom, and venom was detected in only one of the repeat urine samples.

There were insufficient wound aspirates and concentrated urines to be tested by counter-current immunoelectrophoresis against all antisera. Nevertheless, 44 samples positive on immunodiffusion were tested by counter-current immunoelectrophoresis against the appropriate antiserum, and $\mathbf{4 0}$ gave positive results.

\section{Discussion}

We were able to achieve species diagnosis in only $40 \%$ of our patients with snake-bite poisoning, but these results could be improved by the production of better antisera and the use of more sensitive tests than immunodiffusion. For this study antisera were raised in two or three rabbits using crude venom obtained from a single specimen of the appropriate snake species. Identification of the venom component present in biological fluids such as urine and the production of potent antisera against these specific components should improve the success rate of immunodiagnosis. Our inability to show venom in the urine of patients bitten by $E$.carinatus was probably owing to the use of an inappropriate antiserum rather than to failure of patients to excrete venom by this route as we have found labelled protein in the urine of two rabbits injected with ${ }^{125}$ I-labelled $E$. carinatus venom (unpublished).

Immunodiffusion is a slow and insensitive method for detecting antigen and we tried to show venom in samples from some of our patients by other methods. Out of 44 samples positive on immunodiffusion $\mathbf{4 0}$ were also positive on counter- current immunoelectrophoresis, a simple techrique which gives a rapid answer. Counter-current immunoelectrophoresis requires a sample of sufficient volume to fill several immunodiffusion wells, in contrast to immunodiffusion in which a small sample placed in a central well can be tested against several antisera. The small volumes of wound aspirate and concentrated urine available prevented us from using our routine method of counter-current immunoelectrophoresis as the standard test in this study. We have since miniaturized our counter-current technique and can test small volumes of wound aspirate or concentrated urine against the five antisera in routine use. Positive results have been obtained within $\mathbf{3 0}$ minutes in patients bitten by $N$. nigricollis. Counter-current immunoelectrophoresis is the most promising approach to routine immunodiagnosis of snake bite. Latex particles coated with $\gamma$-globulin fractions of specific antisera proved to be very sensitive in detecting purified venom, but false positive and unreadable results were often obtained when wound aspirates and urine samples were tested.

Using a double antibody radioimmunoassay we were able to detect very small amounts of $E$. carinatus venom $(1 \mathrm{ng} / \mathrm{ml})$ in an experimental system (unpublished). Unfortunately, we were unable to apply this assay successfully to the study of serum from patients with snake bite as we often obtained non-specific inhibition with sera from control patients. A solid-phase radioimmunoassay has recently been successfully developed by Coulter et al. (1974) for the detection of the venoms of Notechis scutatus (tiger snake) and Demansia textilis (brown snake), however, and venom was detected in the serum of two patients bitten by these snakes.

Immunodiagnosis may have a number of important roles to play in the study of snake bite. In most geographical areas only a few species of snake are medically important and only a few antisera would be required. A rapid method of immunodiagnosis should be useful to clinicians in deciding which antivenom to give a bitten patient. At present counter-current immunoelectrophoresis offers the most promising approach to this aspect of immunodiagnosis. Immunodiagnosis can also be invaluable as a means of accurate retrospective diagnosis, thus facilitating epidemiogological studies and the compilation of distinctive clinical patterns in poisoning caused by different types of snakes. Radioimmunoassay may prove to be especially valuable in these types of studies. Monitoring of venom levels in serum and urine should also prove to be of value in assessing 
the efficacy of new antivenoms and in determining their effective dosage.

We thank Dr. N. Veall for his help with the radioimmunoassay. This study was supported by grants from the United Kingdom Medical Research Council and from the Wellcome Trust.

\section{References}

Coulter, A. R., Sutherland, S. K., and Broad, A. J. (1974). Fournal of Immunological Methods, 4, 297.

Greenwood, B. M., and Whittle, H. C. (1974). Clinical and Experimental Immunology, 16, 413. Muelling, R. J., Samson, R. F., and Beven, T. (1957). American fournal of
Clinical Pathology, 28, 489.

Trethewie, E. R., and Thomas, W. L. (1969). Toxicon, 7, 243.

\title{
Effect of Age and Menopausal Status on Estimates of Oestrogen Binding by Human Malignant Breast Tumours
}

\author{
H. BRAUNSBERG, V. H. T. JAMES, C. W. JAMIESON, S. DESAI, \\ A. E. CARTER, M. HULBERT
}

British Medical fournal, 1974, 4, 745-747

\section{Summary}

The uptake of oestradiol by human breast-tumour tissue estimated by in-vivo and in-vitro techniques has been examined in relation to patients' ages and menopausal status. Results from in-vivo studies showed no convincing correlations, while in-vitro results were significantly correlated with menopausal status. There was a significant correlation between results obtained by the two techniques.

\section{Introduction}

After claims that the presence of oestrogen-binding components ("receptors") in human malignant breast tumours may be related to their response to endocrine therapy (Jensen et al., 1973; Maass et al., 1972) much work has recently been published on the quantitative determination of these substances. Though estimates of tumour affinity for oestrogen by an in-vivo tracer infusion technique (Braunsberg et al., 1967) may yield results which are independent of plasma oestrogen levels in-vitro methods involving incubation of tracer with tissue extracts (Korenman and Dukes, 1970) are believed to estimate free-that is, unoccupied-oestrogen binding sites because the endogenous hormone dissociates only slowly from its complex (Feherty et al., 1971). It is therefore possible that the estimated concentrations of "receptor" and the incidence of "receptorpositive" tumours depend on plasma oestrogen levels and thus on the patient's age and menopausal status.

Some studies support this possibility (Maass et al., 1972; Feherty et al., 1971; Savlov et al., 1974; Spaeren et al., 1973). We report here a statistical analysis of results for 105 tumours

\footnotetext{
Department of Chemical Pathology, St. Mary's Hospital Medical School, London W2 1PG

H. BRAUNSBERG, PH.D., M.R.C.PATH., Lecturer

V. H. T. JAMES, D.SC., M.R.C.PATH., Professor
}

Surgical Unit, St. Mary's Hospital Medical School, London W2 1PG C. W. JAMIESON, M.s., F.R.C.S., Consultant Surgeon (Present address: St. Thomas's Hospital, London S.E.1)

S. DESAI, F.R.C.S., Lecturer

King Edward Memorial Hospital, Ealing, London W.13

A. E. CARTER, F.R.C.S., Consultant Surgeon

Department of Radiotherapy, St. Mary's Hospital, London, W.2

M. HULBERT, D.M.R.E., F.F.R., Consultant Radiotherapist studied in-vivo (Braunsberg et al., 1967) and 76 tumours studied by an in-vitro technique (Feherty et al., 1971).

\section{Patients and Methods}

Patients were grouped according to their age or their menopausal status; group 1 were premenopausal, group 2 were symptomatically menopausal, group 3 had been postmenopausal for a period up to and including five years, and group 4 had been postmenopausal for more than five years. All tumours were primary lesions whose malignancy was confirmed histologically.

In the in-vivo study the 105 patients were infused with tritium-labelled oestradiol at a constant rate for at least three hours before and during surgery. Under these conditions equilibrium is approached in the tissues between isotopic oestrogen and the endogenous exchangeable hormone. Tissue and plasma obtained at operation were extracted (Braunsberg et al., 1967) to yield the free steroid moiety, and the concentration of radioactive steroid in the tissue was a measure of the concentration of endogenous hormone present (Braunsberg and James, 1967). Oestrogen uptake was expressed as the ratio of radioactivity per gram of tissue $(T)$ to that per millilitre of plasma (P).

For the in-vitro study the method of Feherty et al., (1971) was used. Tissue extracts were incubated with varying amounts of tritium-labelled oestradiol which attaches to binding sites if present. Subsequent incubation with dextran-coated charcoal removed free oestradiol and dissociated the steroid from lowaffinity sites leaving that bound to high-affinity "receptor" in solution. Determination of bound radioactivity at different concentrations of tracer yielded estimates of binding sites.

\section{Results}

For both the in-vivo and the in-vitro studies the logarithms of the values estimated gave approximately Gaussian frequency distributions. All Student's $t$ tests were therefore applied to logarithmic data.

The results for $T: P$ (in-vivo technique) according to patients' ages are shown in fig 1 . There was no significant difference between the age groups. The data plotted according to menopausal status are shown in fig. 2. No significant differences were found between the groups, but when groups 1,2 , and 3 were combined the mean $\mathrm{T}: \mathrm{P}(2.1)$ was just significantly different from that (3.2) for group $4(P<0.05)$.

In the in-vitro study 58 out of 76 tumours $(76 \%)$ were found to bind oestradiol-that is, to be "positive." The incidence of 